Case Reports

\title{
Cryptococcal Meningoencephalitis in a Patient with Hyper IgM Syndrome Due to CD40 Deficiency: Case Report and Literature Review
}

\author{
${ }^{1}$ Maram Al-Banyan, ${ }^{1}$ Abdulhadi Alqahtani, ${ }^{1}$ Farrukh Sheikh, ${ }^{1,2}$ Agha M. Rehan Khaliq, \\ ${ }^{1}$ Hasan Al Rayes, ${ }^{3}$ Ashraf Al-Tarifi, ${ }^{2,4}$ Bandar Al-Saud and ${ }^{1,2}$ Rand K. Arnaout \\ ${ }^{1}$ Department of Medicine, Section of Allergy and Immunology, \\ King Faisal Specialist Hospital and Research Centre, PO Box 3354, Riyadh 11211 Kingdom of Saudi Arabia \\ ${ }^{2}$ Alfaisal University, Riyadh, Kingdom of Saudi Arabia \\ ${ }^{3}$ Department of critical Care Medicine, King Faisal Specialist Hospital and Research Centre, \\ Riyadh, Kingdom of Saudi Arabia \\ ${ }^{4}$ Department of Pediatrics, Section of Allergy and Immunology, \\ King Faisal Specialist Hospital and Research Centre, Riyadh, Kingdom of Saudi Arabia
}

\section{Article history}

Received: 28-11-2018

Revised: 22-02-2019

Accepted: 21-02-2019

Corresponding Author:

Maram Al-Banyan

Department of Medicine,

Section of Allergy and

Immunology, King Faisal Specialist

Hospital and Research Centre, PO

Box 3354, Riyadh 11211, Saudi

Arabia

Tel: +966114427492

Fax: +966114427499

Email: maalbanyan@kfshrc.edu.sa
Abstract: Hyper-IgM syndrome (HIGM) due to CD40 deficiency is a very rare form of combined immunodeficiency with increased susceptibility to opportunistic infections. Cryptococcus is an opportunistic infection usually affecting immunocompromised individuals. This is the first report to describe a patient with HIGM due to CD40 deficiency presenting with meningoencephalitis secondary to Cryptococcus infection.

Keywords: Hyper-IgM Syndrome, Cryptococcus Neoformans, Meningoencephalitis, Primary Immunodeficiency

\section{Introduction}

The prevalence of HIGM varies in different ethnicities around the world.

Globally, all forms of HIGM constitute 0.3-2.9\% of all patients with primary immune deficiencies (Yazdani et al., 2018).

HIGM due to CD40 deficiency is an autosomal recessive inheritance genetic deficit which results in the lack of CD40 expression on the surfaces of B cells and antigen-presenting (Ferrari et al., 2001) Other types of HIGM syndrome include; X-linked HIGM syndrome due to CD40 ligand (CD40L)mutation on activated $\mathrm{T}$ lymphocytes, intracellular deficitsintrinsic to only $\mathrm{B}$ cells, such as autosomal recessive mutations in activation-induced cytidine deaminase (AID) or uracil$\mathrm{N}$-glycosylase (UNG) (Lougaris et al., 2005). Patients affected with HIGM syndrome due to CD40 deficiency have a defective immunoglobulin isotype classswitching recombination (CSR) and Somatic Hyper Mutation (SHM) pathways as well as abnormality in $\mathrm{T}$ cell function. Patients with HIGM due to CD40 or
CD40Ldeficiency usually have a high rate of morbidity and mortality compared to other form of HIGM syndrome. Patients present in infancy with recurrent bacterial, viral and opportunistic infections, as well as protracted diarrhea, failure to thrive and severe neutropenia. In previous large series for patients with HIGM due to CD40 deficiency, one third had Pneumocystis Jiroveci Pneumonia (PJP) and half had chronic diarrhea due to Cryptosporidium and devastating sclerosing cholangitis (Al-Saud et al., 2013). This susceptibility to opportunistic infections in HIGM syndrome due to CD40 deficiency is the results of functional defects in the dendritic cells of patients due to the lack of CD40-CD40L interaction between dendritic cells and activated Tcells, which leads to a defect in Tcell priming and interferon- $\gamma$ secretion.

Cryptococcus neoformans is a spherical, encapsulated, non-myceliated, non-fermenting fungal cell. Okagaki et al. (2010) which can cause severe and fatal meningitis primarily in immunosuppressed humans like HIV patients (Cogliati, 2013). Cryptococcus neoformans most often targets the pulmonary or the 
central nervous system, but other systems can also be infected. Symptoms of cryptococcal meningoencephalitis typically begin indolently. The most common symptoms of cryptococcal infection are fever, fatigue, uneasiness and headache (van Spil et al., 2015). In addition, patients may have confusion, focal neurological deficits or altered mental status.

\section{Case Report}

A 27-year-old Saudi malepatient, teacher by profession, lives in the capital, Riyadh. The patient is known to have HIGM syndrome due to CD40 deficiency on monthly intravenous immunoglobulin and sulfamethoxazole-trimethoprim prophylaxis. The patient's clinical and molecular genetics details was previously reported (3). The patient was in his usual health condition until he presented to our emergency room with intractable headache for the last 10 days. The Headache started gradually, reaching up to $9 / 10$ pain intensity, worse at night, radiating to the neck, aggravated by loud noise and light and associated with blurred vision and photophobia. The headache was poorly responding to oral analgesics. There was no history of associated vomiting, fever, seizures, decreases level of consciousness or any other neurological deficit. There were also no other systemic manifestations. On physical examination, the patient was alert and oriented. Initial neurological examination was normal with intact cranial nerves and normal sensation, power and reflexes. No other systemic physical abnormality was detected.
Initial blood workup revealed hemoglobin of $15.1 \mathrm{~g} / \mathrm{dL}$ (normal: 135-180); leucocytes of $8.78 \times 10^{9} / \mathrm{L}$ (normal: 3.9-11.00) (neutrophils 83\%; lymphocytes 13\%, eosinophils: $3 \%$ ); and platelets of $286 \times 10^{9} / \mathrm{L}$ (normal: 155-435). Blood chemistry showed normal renal and hepatic function and an elevated C-reactive protein level of $14.5 \mathrm{mg} / \mathrm{L}$ (normal: $<3 \mathrm{mg} / \mathrm{L}$ ). The Computed Tomography (CT) scan of the patient's brain showed mild effacement of the cerebral sulci with reduced white matter CT density (Fig. 1), suggestive of diffuse brain edema. The patient initially refused Lumbar Puncture (LP) for Cerebrospinal Fluid (CSF) analysis and culture. Therefore, the team empirically treated for meningoencephalitis with Acyclovir, Ceftriaxone and Vancomycin. No anti-fungal treatment was started butfungitelldid come back positive and blood Cryptococcal antigen came back negative. Despite the initiation of anti-virals and antibiotics the patient started to become agitated and confused. He was reassessed by neurology and found to have bilateral 6th nerve palsy and papilledema. So the patient was electively intubated and admitted to the intensive care unit. A multiplanar multi sequential brain MRI without and with contrast administration of the brain was done and showed mild brain swelling and bilateral flattening of the optic nerve heads suggestive of papilledema (Fig. 2). With the deterioration in his condition LP was performed and CSF extracted. CSF analysis showed clear fluid with WBC:272, neutrophils:9, lymphocytes:76, Eosinophils:6 Gram stain was negative, India ink stain showed encapsulated yeast organisms.

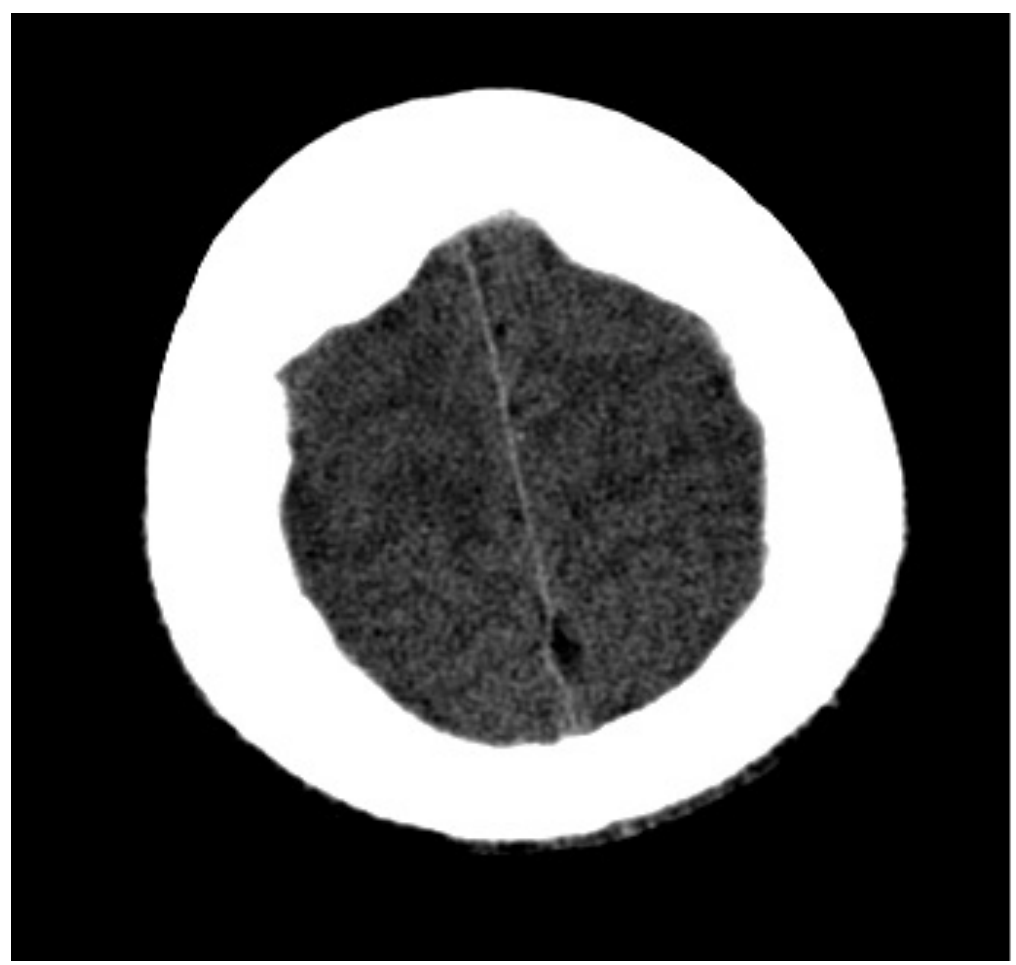

Fig. 1: Mild effacement of the cerebral sulci with reduced white matter CT density, suggestive of diffuse brain edema 

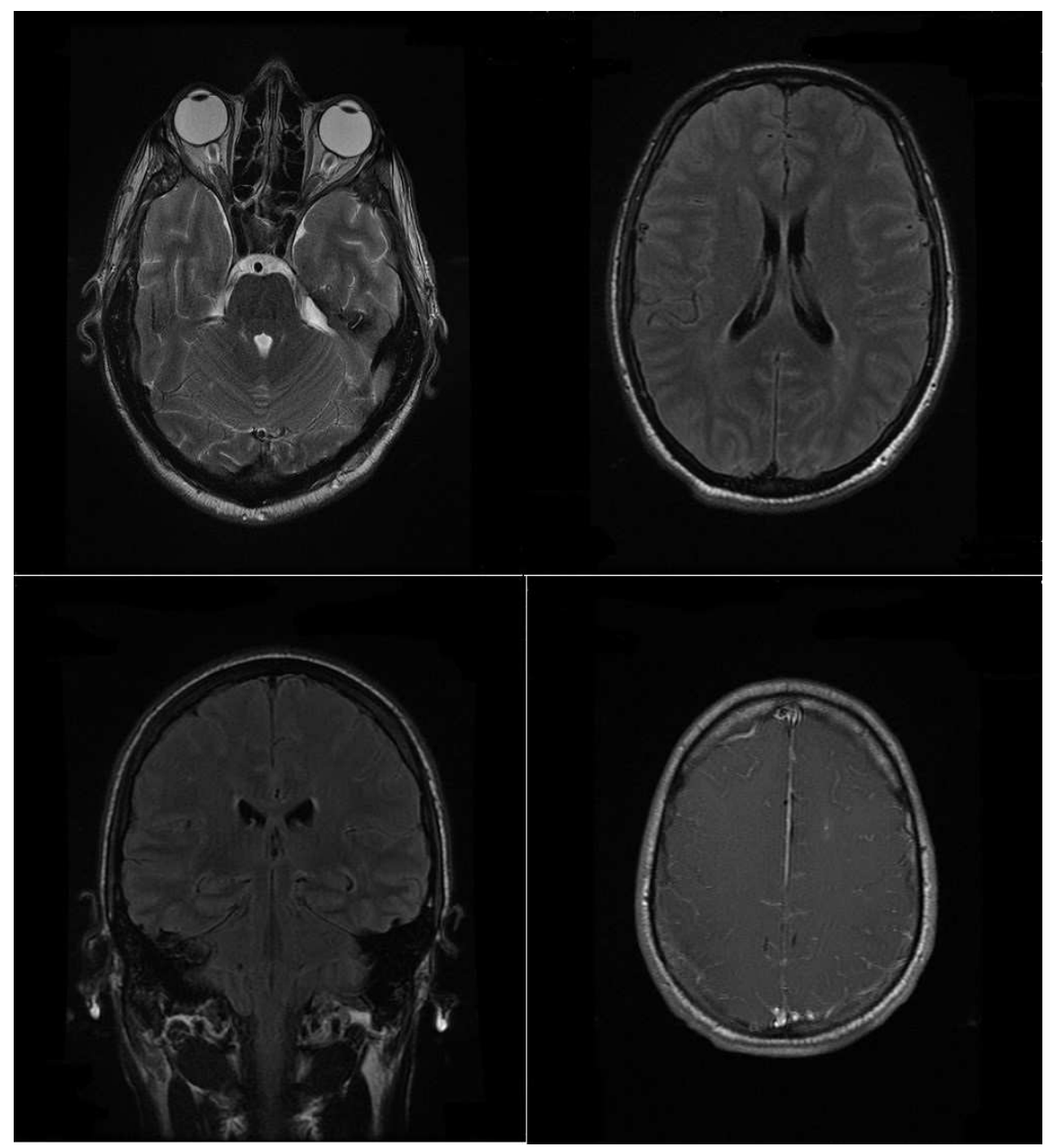

Fig. 2: MRI of the brain showing mild brain swelling and bilateral flattening of the optic nerve heads suggestive of papilledema

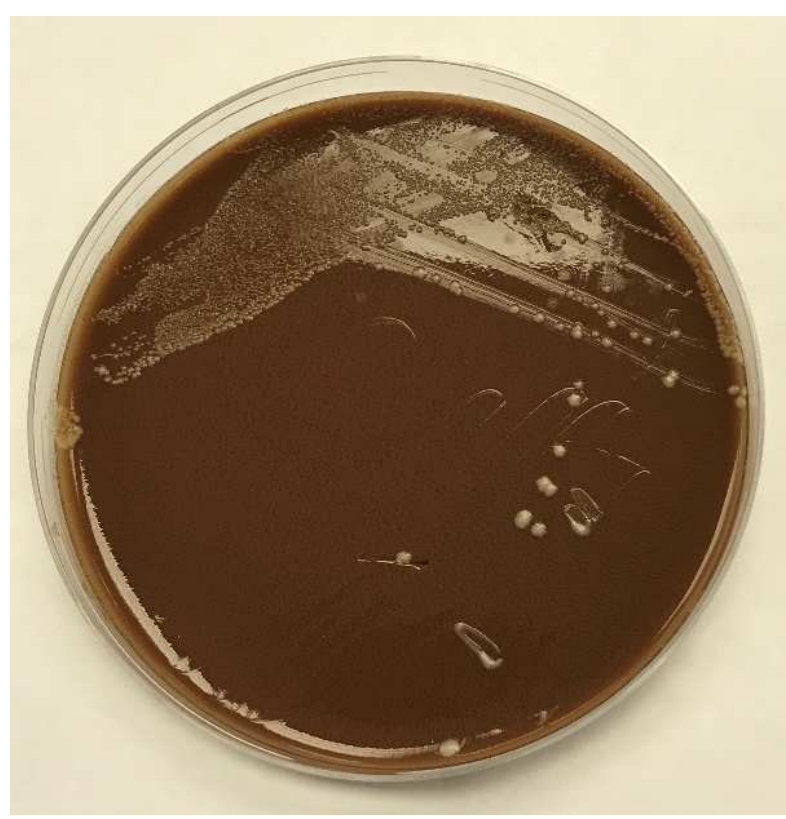

Fig. 3: Chocolate agar growing Cryptococciin our patien 
CSF Culture: showed moderate Cryptococcus neoformans. The repeated blood culture using BACTAC culture system grew: Cryptococci neoformans. A chocolate agar culture of the CSF revealed grayish colonies, consistent with Cryptococcus spp. (Fig. 3). The patient was immediately started on Amphotericin B and Flucytosine. After a few days on treatment the patient's symptoms resolved. Due to positive blood culture for cryptococcus, treatment had to be continued for a total of four weeks on Amphotericin B and Flucytosine. Then prophylaxis of Fluconazole was continued until the patient received stem cell transplant as a final cure for his immune deficiency (Fontana et al., 2003).

\section{Discussion}

Our patient was diagnosed with CD40 deficiency a form of Combined Immune Deficiency (CID) which is well known to be associated with opportunistic infections like Pneumocystis Jiroveci Pneumonia (PJP) and others (Al-Saud et al., 2013). Cryptococcal infection is one of these opportunistic infections that we should think of in any patient with combined immune deficiency, although in reality it is not a common occurrence evidenced by the fact that only a few cases have been reported in CID patients of Cryptococcal infections (Winkelstein et al., 2003; Levy et al., 1997; Lee et al., 2001; Jo et al., 2002). Although our patient was compliant to his prophylactic medications and to his followup he still developed this uncommon complication.

Cryptococcal infection classically effects immunocompromised patients or patients with other predisposing factors like end-stage liver disease, renal failure and sarcoidosis (Kiertiburanakul et al., 2006; Yuchong et al., 2012)

However it can also affect patients who are apparently normal. A subpopulation of these otherwise normal patients probably have subclinical immune deficiencies (Ecevit et al., 2006).

To the best of our knowledge, this is the first reported case of cryptococcal meningitis in a patient with HIGM due to CD 40 deficiency. One other case has previously been reported in a patient with HIGM due to CD40 L deficiency (Malheiro et al., 2014).

The clinical presentation of thepreviously reported patient was similar to ours.

In our patient the symptoms were indolent as he presented with headache and malaise for two weeks which started gradually and associated with photophobia and blurred vision but there was no focal neurological deficitinitially. A cerebral CT scan was done for him and showed mild effacement of the cerebral sulci with reduced white matter CT density, suggestive of diffuse brain edema.

Despite the fact that there was no abnormality in his blood that suggested an infection, LP was done based on high clinical suspicion. The lack of symptoms initially may be due to the compromised immune response to Cryptococcus spp as it was shown in cases of CD40 deficiency that in vitro the CD154-CD40 interaction is essential for the secretion of IL-12 and IFN $\gamma$ in response to this organism (Grewal et al., 1995)

Macrophages need IL-12 and IFN $\gamma$ cytokines to activate T-cells through antigen-presenting dendritic cells. With these immunological abnormalities, patients with CD40 deficiency will be more susceptible to fungal infection since there isaltered generation of TH1 cells that are normally responsible for stimulating phagocytic activity towards fungal infections. As a consequence, the immune reaction will be less severe, with reduced immune activation and migration and fewer symptoms that are typically associated with meningeal inflammation (Malheiro et al., 2014)

Current guidelines for cryptococcal treatment recommendstarting Amphotericin B and Flucytosine. In our case, these were effective therapies against Cryptococcus neoformans evidenced by the clinical improvement and the negative LP after completion of treatment (Perfect et al., 2010). Although this treatment was effective, there is no consensus on the appropriate duration for maintenance therapy. The previously reported HIGM patients with this infection were treated similar to HIV patients with cryptococcal infection (Lee et al., 2001; Jo et al., 2002; Malheiro et al., 2014). In the HIV setting, there is evidence that maintenance therapy should be kept until an immunologic improvement, characterized by CD4+ T-cells $>100 / \mu \mathrm{L}$ for more than 3 months, is achieved (Levy et al., 1997). In our case immune reconstitution will only happen after the patient receives stem cell transplantation.

Due to lack of data on the duration of antifungal therapy in patients similar to ours; and the fact that our patient refused future hematopoietic stem cell transplant, we put our patient on prophylactic therapy with lifelong fluconazole, with the need to monitor and prevent any future drug toxicity.

\section{Conclusion}

Patients with Hyper-IgM syndrome (HIGM) are more proneto all types of infections, including, bacterial, fungal and viral infections and need prophylaxis therapy in addition to close monitoring and follow-up.

Our case report demonstrates that; although cryptococcus is a common organism effecting the severely immunocompromised it has only been previously reported in one patient with HIGM CD 40L deficiency, Making this the first case in HIGM CD40 deficiency organisms like Cryptococcus spp. We encourage close monitoring for treatment response and high grade of clinical suspicion and a wider differential 
diagnosis even for rare complications in patients with primary immune deficiency.

\section{Acknowledgement}

We would like to thank the departments of microbiology and radiology for their contribution.

\section{Author's Contributions}

Maram Al-Banyan: Designed and wrote the manuscript.

Abdul HadiAlQahtani: Designed and wrote the manuscript.

Farrukh Sheikh: Wrote and reviewed the manuscript.

Agha M. Rehan Khaliq: Reviewed the manuscript.

Hasan Al Rayes: Reviewed the manuscript.

Ashraf Al-Tarifi: Reviewed the manuscript.

Bandar Al-Saud: Wrote and reviewed the manuscript.

Rand K. Arnaout: Wrote the manuscript and gave final approval.

\section{Ethics}

This article is original and contains unpublished material. The corresponding author confirms that all of the other authors have read and approved the manuscript and no ethical issues involved.

\section{References}

Al-Saud, B.K., Z. Al-Sum, H. Alassiri, A. Al-Ghonaium and S. Al-Muhsen et al., 2013. Clinical, immunological and molecular characterization of hyper-IgM syndrome due to CD40 deficiency in eleven patients. J. Clin. Immunol., 33: 1325-1335.

Cogliati, M., 2013. Global molecular epidemiology of cryptococcus neoformans and cryptococcus gattii: An atlas of the molecular types. Scientifica, 2013: 675213.

Ecevit, I.Z., C.J. Clancy, I.M. Schmalfuss and M.H. Nguyen, 2006. The poor prognosis of central nervous system cryptococcosis among nonimmunosuppressed patients: A call for better disease recognition and evaluation of adjuncts to antifungal therapy. Clin Infect. Dis., 42: 1443-1447.

Ferrari, S., S. Giliani, A. Insalaco, A. Al-Ghonaium and A.R. Soresina et al., 2001. Mutations of CD40 gene cause an autosomal recessive form of immunodeficiency with hyper IgM. Proc. Natl. Acad Sci. USA, 98: 12614-12619.

Fontana, S., D. Moratto, S. Mangal, M. De Francesco and W. Vermi, 2003. Functional defects of dendritic cells in patients with CD40 deficiency. Blood, 102: 4099-4106.
Grewal, I.S., J. Xu and R.A. Flavell, 1995. Impairment of antigen-specific T-cell priming in mice lacking CD40 ligand. Nature, 378: 617-620.

Jo, E.K., H.S. Kim, M.Y. Lee, M. Iseki and J.H. Lee et al., 2002. X-linked hyper-IgM syndrome associated with Cryptosporidium parvum and Cryptococcus neoformans infections: the first case with molecular diagnosis in Korea. J. Korean Med. Sci., 17: 116-120.

Kiertiburanakul, S., S. Wirojtananugoon, R. Pracharktam and S. Sungkanuparph, 2006. Cryptococcosis in human immunodeficiency virus-negative patients. Int. J. Infect. Dis., 10: 72-78.

Lee, M.Y., J.H. Chung, J.H. Shin, T.J. Hwang and K.S. Kim et al., 2001. Lymphonodular cryptococcosis diagnosed by fine needle aspiration cytology in hyper-IgM syndrome: A case report. Acta Cytol., 45: 241-244.

Levy, J., T. Espanol-Boren, C. Thomas, A. Fischer and P. Tovo et al., 1997. Clinical spectrum of X-linked hyper-IgM syndrome. J. Pediatr., 131: 47-54.

Lougaris, V., R. Badolato, S. Ferrari and A. Plebani, 2005. Hyper immunoglobulin $M$ syndrome due to CD40 deficiency: Clinical, molecular and immunological features. Immunol. Rev., 203: 48-66.

Malheiro, L., D. Lazzara, S. Xerinda, M.D. Pinheiro and A. Sarmento, 2014. Cryptococcal meningoencephalitis in a patient with hyper immunoglobulin M (IgM) syndrome: A case report. BMC Res. Notes, 7: 566.

Okagaki, L.H., A.K. Strain, J.N. Nielsen, C. Charlier and N.J. Baltes et al., 2010. Cryptococcal cell morphology affects host cell interactions and pathogenicity. PLoS Pathog., 6: e1000953.

Perfect, J.R., W.E. Dismukes, F. Dromer, D.L. Goldman and J.R. Graybill et al., 2010. Clinical practice guidelines for the management of cryptococcal disease: 2010 update by the infectious diseases society of America. Clin. Infect. Dis., 50: 291-322.

van Spil, W.E., S. Nooijen, P.Y. de Jong, R.P. Aliredjo and R.G. de Sevaux et al., 2015. [Cryptococcal meningitis]. Ned Tijdschr Geneeskd, 159: A8478.

Winkelstein, J.A., M.C. Marino, H. Ochs, R. Fuleihan and P.R. Scholl et al., 2003. The X-linked hyperIgM syndrome: Clinical and immunologic features of 79 patients. Medicine (Baltimore), 82: 373-384.

Yazdani, R., S. Fekrvand, S. Shahkarami, G. Azizi and B. Moazzami et al., 2018. The hyper IgM syndromes: Epidemiology, pathogenesis, clinical manifestations, diagnosis and management. Clin Immunol., 198: 19-30.

Yuchong, C., C. Fubin, C. Jianghan, W. Fenglian and X. Nan et al., 2012. Cryptococcosis in China (19852010): Review of cases from Chinese database. Mycopathologia, 173: 329-335. 